\title{
Relation of Maximum Lifetime Body Mass Index with Age at Hemodialysis Initiation and Vascular Complications in Japan
}

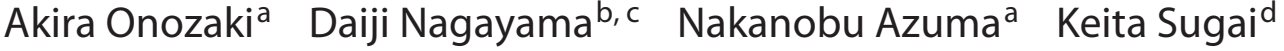 \\ Etsuko Shitarad $^{d}$ Takehiko Sakai $^{\mathrm{e}}$ Motoyuki Masai $^{\mathrm{e}}$ Kohji Shiraif \\ Ichiro Tatsunoc, g
}

a'nternal Medicine, Tokatsu Clinic Hospital, Chiba, Japan; 'b Internal Medicine, Nagayama Clinic, Tochigi, Japan; ${ }^{c}$ Center of Diabetes, Endocrine and Metabolism, Toho University Sakura Medical Center, Chiba, Japan; ${ }^{\text {N Nutrition }}$ Management Division, Mihama Hospital, Chiba, Japan; 'Department of Urology, Mihama Hospital, Chiba, Japan; fInternal Medicine, Mihama Hospital, Chiba, Japan; ${ }^{9}$ Chiba Prefectural University of Health Sciences, Chiba, Japan

\section{Keywords}

Maximum lifetime body mass index - Obesity management . End-stage renal disease $\cdot$ Hemodialysis initiation - Diabetic retinopathy

\begin{abstract}
Objective: The aim of this study was to investigate the association of the maximum lifetime body mass index (max BMI) with hemodialysis initiation and comorbidities in Japanese hemodialysis patients. Methods: In a retrospective crosssectional study on 724 hemodialysis patients, max BMI, age at hemodialysis initiation, and comorbidities including sleep apnea syndrome, cerebro-cardiovascular diseases, and proliferative diabetic retinopathy (PDR) were analyzed. Early hemodialysis initiation was defined as age $<50$ years. Result: Diabetes patients showed a higher max BMI and prevalence of atherosclerotic diseases than nondiabetes patients, despite almost the same age at hemodialysis initiation. Patients with early hemodialysis initiation showed higher male ratio, prevalence of PDR, and max BMI than those with later initiation, despite almost equal prevalence of diabetes. Receiveroperating characteristic curve analysis determined a max BMI of $28.4 \mathrm{~kg} / \mathrm{m}^{2}$ as a reliable cutoff value for predicting
\end{abstract}

karger@karger.com www.karger.com/ofa

Karger"

BOPEN ACCESS
(C) 2021 The Author(s)

Published by S. Karger AG, Basel

This is an Open Access article licensed under the Creative Common Attribution-NonCommercial-4.0 International License (CC BY-NC) (http://www.karger.com/Services/OpenAccessLicense), applicable to the online version of the article only. Usage and distribution for commercial purposes requires written permission. early hemodialysis initiation, and this parameter was identified as an independent predictor of early hemodialysis initiation using bivariate logistic regression analysis. Vitrectomy for PDR also tended to contribute independently to early hemodialysis initiation. Conclusion: A high max BMI contributed to early hemodialysis initiation independent of diabetes. Furthermore, PDR was associated with a high max BMI and early hemodialysis initiation. These results suggest that weight reduction in young chronic kidney disease patients with obesity may prevent hemodialysis and blindness.

(c) 2021 The Author(s)

Published by S. Karger AG, Basel

\section{Introduction}

Overweight and obesity are defined as abnormal or excessive fat accumulation that may impair health and have been reported to be associated with glucose intolerance, dyslipidemia, and hypertension, thus increasing cardiovascular risks [1-3]. Furthermore, the epidemic of obesity parallels the increase in incidence of chronic kidney

Akira Onozaki and Daiji Nagayama contributed equally to this work.
Correspondence to:

Daiji Nagayama, deverlast96071@gmail.com 
disease (CKD). Several recent studies have shown that obesity is an independent predictor of CKD and endstage renal disease (ESRD) that requires either hemodialysis or kidney transplant [4]. Obesity is closely related to the pathogenesis of diabetes, and diabetic nephropathy is now the leading cause of hemodialysis initiation. Furthermore, diabetic retinopathy is the principal cause of acquired vision impairment worldwide [5]. In fact, many hemodialysis patients suffer from blindness as well as cerebro-cardiovascular disease. Since the burden of obesity has increased globally, therapeutic intervention for obesity to reduce hemodialysis initiation and vision impairment is needed.

In an analysis using data from the National Health and Nutrition Examination Survey, Mexican American adults whose maximum lifetime body mass index (max BMI) $\geq 30 \mathrm{~kg} / \mathrm{m}^{2}$ had increased risk of all-cause mortality, even if they were already nonobese at survey [6]. Thus, in addition to the current BMI status, max BMI may also be considered an important risk factor for ESRD and blindness. However, it remains controversial whether obesity promotes CKD independent of the presence of diabetes. In addition, it is not fully verified how a past history of obesity acts as a risk for vascular complications even if obesity is later alleviated.

In this retrospective multicenter cross-sectional study, we investigated how the max BMI is involved in hemodialysis initiation and the comorbidities including diabetic retinopathy. Furthermore, the potential confounding risk factors for early hemodialysis initiation were also examined.

\section{Materials and Methods}

\section{Study Design}

This multicenter, retrospective cross-sectional study was conducted in 2 specialized hemodialysis centers of Tokatsu Clinic Hospital (Matsudo, Japan) and Mihama Hospital (Chiba, Japan). Patients who gave written informed consent were recruited in the study. A total of 926 patients initiated hemodialysis in the 2 centers between June 1, 2010, and May 31, 2016. From these patients, 724 who gave informed consent were included in the study. All participants were Japanese, and no other ethnically different populations were included.

\section{Data Collection}

The following data were extracted from the medical records: height, body weight at the age at hemodialysis initiation, maximum body weight of lifetime, and comorbidities including prior atherosclerotic diseases (myocardial infarction, coronary revascularization, percutaneous transluminal angioplasty for peripheral artery disease [PAD], limb amputation, and cerebral stroke), sleep apnea syndrome (SAS), and invasive treatment for proliferative diabetic retinopathy (PDR) (laser photocoagulation and vitrectomy surgery). Coronary revascularization included percutaneous coronary intervention and coronary artery bypass grafting. Cerebral stroke was defined as tissue evidence of cerebral infarction or intracranial bleeding diagnosed by X-ray computed tomography or magnetic resonance imaging associated with symptoms lasting longer than $24 \mathrm{~h}$. SAS was defined by the use of continuous positive airway pressure. A combination of $\geq 6.5 \%$ HbA1c with $\geq 126$ $\mathrm{mg} / \mathrm{dL}$ fasting plasma glucose was adopted to detect diabetes [7]. Participants receiving antidiabetic agents were also diagnosed as diabetes.

BMI $\left(\mathrm{kg} / \mathrm{m}^{2}\right)$ was calculated as weight $(\mathrm{kg})$ divided by the square of height $(\mathrm{m})$. We adopted dry weight determined for calculating at hemodialysis initiation. According to the diagnostic criteria for obesity by Japan Society for the Study of Obesity, obesity was defined as BMI $\geq 25 \mathrm{~kg} / \mathrm{m}^{2}$ [8]. Early hemodialysis initiation was defined as initiation before age 50 years (age 50 hereinafter).

\section{Statistical Analysis}

The data are expressed as mean \pm standard deviation (SD) or percentage. For comparison of 2 independent groups, all data were analyzed using Mann-Whitney U-test or Fisher's exact test. Pearson's product-moment correlation coefficient (R) was used to examine the relationship between the max BMI and age at hemodialysis initiation in all participants. Sensitivity and specificity with respect to hemodialysis initiation before age 50 were analyzed using conventional receiver-operating characteristic (ROC) curves. To compare clinical characteristics between tertiles of the max BMI, ANOVA was used for continuous variables and CochranArmitage method for categorical variables. Subsequently, the Bonferroni method was used to compare between lower (T1) and upper (T3) tertile groups. Bivariate logistic regression analyses were performed to identify the contributors to hemodialysis initiation before age 50 years, and the result was expressed as odds ratio with $95 \%$ confidence interval. A 2 -sided $p$ value of 0.05 was considered statistically significant. Statistical analyses were performed using EZR (version 1.40; Saitama Medical Center, Jichi Medical University, Saitama, Japan) [9].

\section{Results}

\section{Comparison of Characteristics of Hemodialysis}

Patients with and Those without Diabetes

In the 724 participants, the most common cause of hemodialysis initiation was diabetic nephropathy (48.2\%), followed by chronic glomerulonephritis (18.7\%), nephrosclerosis (14.0\%), and polycystic kidney (5.4\%), while $13.8 \%$ had other or unknown causes. This distribution of causes is consistent with the annual hemodialysis data for 2018 reported by Japanese Society for Hemodialysis Therapy Renal Data Registry [10]. Note that not all diabetes patients started hemodialysis due to diabetic nephropathy. 
Table 1. Comparison of demographic and clinical characteristics of hemodialysis patients with or without diabetes

\begin{tabular}{lcccc}
\hline Variables & All & Nondiabetes & Diabetes \\
$(N=724)$ & $(N=332)$ & $p$ value \\
& 72.2 & 66.0 & 77.6 & $<0.001^{*}$ \\
Male, \% & $65.2 \pm 13.2$ & $66.1 \pm 14.1$ & $64.4 \pm 12.4$ & 0.037 \\
Age, years & $62.7 \pm 13.3$ & $63.5 \pm 14.1$ & $61.9 \pm 12.5$ & 0.052 \\
Age at dialysis initiation, years & 20.0 & 19.6 & 20.4 & $0.852^{*}$ \\
Age at dialysis initiation $<50$ years, \% & $2.6 \pm 1.7$ & $2.6 \pm 1.7$ & $2.5 \pm 1.7$ & 0.386 \\
Period of dialysis, years & $22.8 \pm 3.9$ & $21.8 \pm 3.6$ & $23.6 \pm 3.9$ & $<0.001$ \\
BMI at dialysis initiation, kg/m ${ }^{2}$ & 1.4 & 1.2 & 1.5 & $0.761^{*}$ \\
BMI at dialysis initiation $\geq 35 \mathrm{~kg} / \mathrm{m}^{2}, \%$ & $28.0 \pm 5.2$ & $26.2 \pm 4.9$ & $29.5 \pm 5.0$ & $<0.001$ \\
Max BMI, kg/m ${ }^{2}$ & 9.5 & 4.5 & 13.8 & $<0.001^{*}$ \\
Max BMI $\geq 35$ kg/m ${ }^{2}$ \% & $5.2 \pm 3.6$ & $4.4 \pm 3.3$ & $5.9 \pm 3.8$ & $<0.001$ \\
BMI difference, kg/m ${ }^{2}$ & 5.2 & 2.7 & 7.4 & $0.007^{*}$ \\
Sleep apnea syndrome, \% & 34.4 & 13.9 & 51.8 & $<0.001^{*}$ \\
Prevalence of atherosclerotic diseases, \% & 7.2 & 4.2 & 9.7 & $0.006^{*}$ \\
$\quad$ Myocardial infarction, \% & 23.3 & 4.2 & 39.5 & $<0.001^{*}$ \\
Coronary revascularization, \% & 5.2 & 2.1 & 7.9 & $<0.001^{*}$ \\
Percutaneous transluminal angioplasty for PAD, \% & 2.9 & 0.6 & 4.8 & $<0.001^{*}$ \\
$\quad$ Limb amputation, \% & 10.8 & 9.0 & 12.5 & $0.152^{*}$ \\
Cerebral stroke, \% & - & - & 52.6 & - \\
PDR, \% & - & - & 31.9 & -
\end{tabular}

Data are presented as mean \pm SD or percentage. Mann-Whitney U test. Max BMI, maximum lifetime body mass index; BMI, body mass index; PAD, peripheral artery disease; SD, standard deviation; PDR, proliferative diabetic retinopathy. * Fisher's exact test was used to compare diabetes and nondiabetes patients.

Fig. 1. Distribution of age at hemodialysis initiation. Open bar indicates female subjects and closed bar indicates male subjects. Data at upper part of the graph: age at hemodialysis initiation expressed in median (interquartile range). ${ }^{*} p=0.005$, MannWhitney U-test.

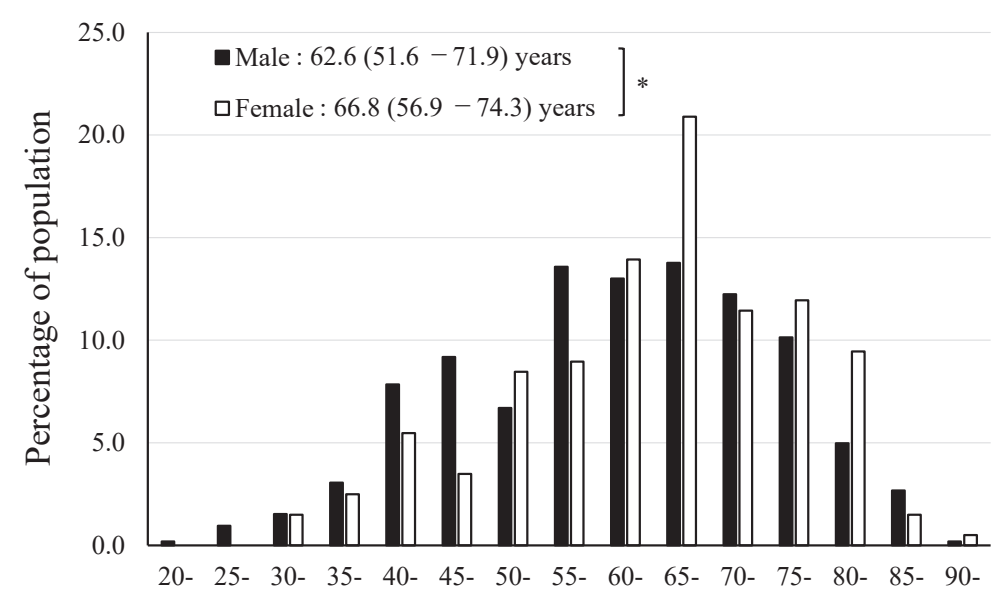

Age at hemodialysis initiation (years)
The comparisons of baseline characteristics and clinical features between diabetes and nondiabetes patients are shown in Table 1 . At the time of this study, $54.1 \%$ of the participants had diabetes, $1.18 \%$ of whom had type 1 , while the remaining had type 2 diabetes. The prevalence of PDR in diabetes patients was $52.6 \%$, including $29.6 \%$ who underwent laser photocoagulation and $12.5 \%$ who had vitrectomy surgery. 
Fig. 2. Distribution of hemodialysis patients in max BMI groups compared to general Japanese population. * Data of Japanese population aged 20-69 years excerpted from the Cross-Sectional Study Using National Health and Nutrition Survey in Japan (2018) (ref. 10). Max BMI, maximum lifetime body mass index; BMI, body mass index.

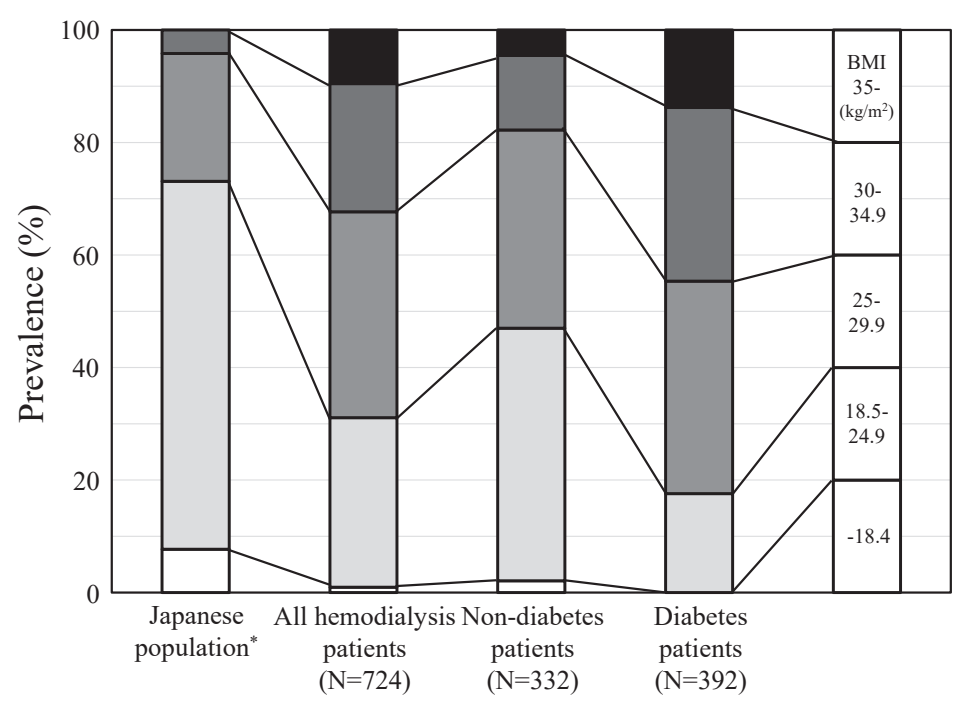

Compared with nondiabetes patients, diabetes patients had a significantly higher male ratio (77.6 vs. $66.0 \%), \mathrm{BMI}$ at hemodialysis initiation (23.6 vs. $21.8 \mathrm{~kg} /$ $\mathrm{m}^{2}$ ), max BMI (29.5 vs. $\left.26.2 \mathrm{~kg} / \mathrm{m}^{2}\right)$, prevalence of max $\mathrm{BMI} \geq 35 \mathrm{~kg} / \mathrm{m}^{2}$ (13.8 vs. $4.5 \%$ ), prevalence of sleep apnea syndrome (7.4 vs. $2.7 \%$ ), and prevalence of prior atherosclerotic diseases (51.8 vs. 13.9\%). On the other hand, no significant difference in age at hemodialysis initiation was observed between 2 groups (61.9 vs. 63.5 years).

\section{Distribution of Age at Hemodialysis Initiation}

Figure 1 shows the distribution of age at hemodialysis initiation by gender. Both genders showed normal distribution. Hemodialysis was initiated at a younger age in men than in women (median: 62.6 vs. 66.9, interquartile range: $51.6-71.9$ vs. $56.9-74.3, p=0.005$ ). In this study, early hemodialysis initiation was conveniently defined as initiation before age 50, and 145 patients $(20.0 \%)$ fulfilled this criterion.

\section{Distribution of Hemodialysis Patients in Max BMI} Groups Compared to General Japanese Population

Distribution of hemodialysis patients in max BMI groups is shown in Figure 2. For comparison with general Japanese population, current BMI data of the population aged 20-69 years were excerpted from the Cross-Sectional Study Using National Health and Nutrition Survey in Japan (2018) [11]. Compared to the standard BMI distribution of Japanese general population, hemodialysis patients in the present study showed a high prevalence of obesity ( $\geq 25 \mathrm{~kg} / \mathrm{m}^{2}$ ) (68.9 vs. $\left.26.9 \%\right)$. Furthermore, a markedly higher prevalence of obesity (82.4\%) was observed in hemodialysis patients with diabetes.

\section{Characteristics of Patients Stratified by Tertiles of}

\section{Max BMI}

Next, all participants were divided according to tertiles of the max BMI (T1: lower, T2: middle, and T3: upper) as shown in Table 2. Compared with the lowest tertile (T1), the highest max BMI tertile (T3) was associated with a higher male ratio, younger age at hemodialysis initiation, and higher prevalence of diabetes, SAS, and atherosclerotic diseases including PDR. Among the atherosclerotic diseases, only the prevalence of ischemic heart disease requiring coronary revascularization showed a significant difference between $\mathrm{T} 1$ and $\mathrm{T} 3$ groups. The prevalence of PDR increased with an increasing max BMI and a similar significant increasing trend was observed with invasive treatment for PDR. In addition, a negative correlation $(R=-0.260, p<0.0001)$ between the max BMI and age at hemodialysis initiation was observed in all participants (data not shown).

\section{Comparison of Characteristics of Hemodialysis}

Patients with Hemodialysis Initiation before and after Age 50

Comparison of early versus later hemodialysis initiation is shown in Table 3. Hemodialysis patients with hemodialysis initiated before age 50 showed a higher male ratio, higher prevalence of SAS, and higher max BMI than 
Table 2. Characteristics of patients stratified by tertiles of max BMI

\begin{tabular}{|c|c|c|c|c|}
\hline \multirow[t]{2}{*}{ Variables } & \multicolumn{3}{|c|}{ Tertile of max BMI } & \multirow{2}{*}{$\begin{array}{l}p \text { value for } \\
\text { trend }\end{array}$} \\
\hline & $\begin{array}{l}\text { lower }(\mathrm{T} 1) \\
(-25.3)\end{array}$ & $\begin{array}{l}\text { middle (T2) } \\
(25.4-29.7)\end{array}$ & $\begin{array}{l}\text { upper (T3) } \\
(29.8-)\end{array}$ & \\
\hline Male, \% & 62.7 & 75.6 & $78.4^{*}$ & $<0.001$ \\
\hline Age, years & $67.1 \pm 13.1$ & $68.5 \pm 11.8$ & $60.0 \pm 13.1^{* *}$ & $<0.001$ \\
\hline Age at dialysis initiation, years & $64.4 \pm 13.1$ & $66.2 \pm 11.9$ & $57.4 \pm 13.2^{* *}$ & $<0.001$ \\
\hline Age at dialysis initiation $<50$ years, $\%$ & 14.5 & 12.8 & $32.8^{*}$ & $<0.001$ \\
\hline Period of dialysis, years & $2.7 \pm 1.7$ & $2.3 \pm 1.7$ & $2.6 \pm 1.7$ & 0.028 \\
\hline BMI at dialysis initiation, $\mathrm{kg} / \mathrm{m}^{2}$ & $20.0 \pm 2.3$ & $22.4 \pm 2.5$ & $25.9 \pm 4.0^{* *}$ & $<0.001$ \\
\hline BMI at dialysis initiation $\geq 35 \mathrm{~kg} / \mathrm{m}^{2}, \%$ & 0.0 & 0.0 & $4.1^{*}$ & $<0.001$ \\
\hline Max BMI, $\mathrm{kg} / \mathrm{m}^{2}$ & $22.7 \pm 1.9$ & $27.5 \pm 1.3$ & $33.7 \pm 3.7^{* *}$ & $<0.001$ \\
\hline Max BMI $\geq 35 \mathrm{~kg} / \mathrm{m}^{2}, \%$ & 0.0 & 0.0 & $28.6^{*}$ & $<0.001$ \\
\hline BMI difference, $\mathrm{kg} / \mathrm{m}^{2}$ & $2.7 \pm 1.9$ & $5.1 \pm 2.4$ & $7.9 \pm 4.1^{* *}$ & $<0.001$ \\
\hline Diabetes, $\%$ & 32.8 & 55.4 & $74.3^{*}$ & $<0.001$ \\
\hline Sleep apnea syndrome, $\%$ & 2.5 & 3.3 & $10.0^{*}$ & $<0.001$ \\
\hline Prevalence of atherosclerotic diseases, $\%$ & 22.0 & 37.2 & $44.0^{*}$ & $<0.001$ \\
\hline Myocardial infarction, \% & 5.4 & 7.4 & 8.7 & 0.158 \\
\hline Coronary revascularization, $\%$ & 12.9 & 23.6 & $33.6^{*}$ & $<0.001$ \\
\hline Percutaneous transluminal angioplasty for PAD, \% & 4.1 & 5.4 & 6.2 & 0.307 \\
\hline Limb amputation, $\%$ & 1.7 & 2.5 & 4.6 & 0.057 \\
\hline Cerebral stroke, $\%$ & 8.3 & 12.4 & 12.0 & 0.189 \\
\hline PDR, \% & 16.2 & 26.0 & 43.2 & $<0.001$ \\
\hline Invasive treatment for diabetic retinopathy, $\%$ & 9.1 & 16.5 & 26.1 & $<0.001$ \\
\hline Laser photocoagulation, $\%$ & 8.7 & 15.3 & 24.1 & $<0.001$ \\
\hline Vitrectomy surgery, $\%$ & 2.9 & 5.4 & 12.0 & $<0.001$ \\
\hline
\end{tabular}

Data are presented as mean $\pm \mathrm{SD}$, or percentage. $p$ value for trend was obtained by ANOVA for continuous variables or by the Cochran-Armitage method for categorical variables. Max BMI, maximum lifetime body mass index; BMI, body mass index; PAD, peripheral artery disease; SD, standard deviation; ANOVA, analysis of variance; PDR, proliferative diabetic retinopathy. ${ }^{*} p<0.001$, T1 versus T3, ANOVA followed by Bonferroni correction. ${ }^{* *} p<0.01$, T1 versus T3, Fisher's exact test followed by Bonferroni correction.

those with later initiation. In addition, the percentage of PDR requiring vitrectomy surgery was significantly higher in patients with early hemodialysis initiation than in those with later initiation, despite almost equal prevalence of diabetes between 2 groups. In contrast, the prevalence of cerebral stroke was higher in patients with hemodialysis initiation at 50 years or later. These variables that showed significant differences in this comparison were used in subsequent logistic regression analyses (Table 4).

\section{Discriminatory Power of Max BMI for Hemodialysis \\ Initiation before Age 50 Years}

To evaluate the discriminatory power and cutoff value of the max BMI for early hemodialysis initiation, an ROC curve was generated and Youden's J Index, which represents the maximum sensitivity + specificity -1 for all cutoff points [12], was calculated. Resultantly, the diagnostic accuracy indicated by area under the ROC curve of the $\max$ BMI for early hemodialysis initiation was 0.653 (95\% CI: $0.599-0.708 ; p<0.001$ ), and the cutoff value of the max BMI was 28.4 (sensitivity: 63.4\%, specificity: $62.5 \%$ ). On the other hand, BMI of $35.0 \mathrm{~kg} / \mathrm{m}^{2}$ has been reported to be the cutoff value of severe obesity indicated for bariatric surgery [13]. These 2 cutoff values were verified in subsequent logistic regression analyses.

\section{Bivariate Logistic Regression Models of the Association between Hemodialysis Initiation before Age 50 Years and Clinical Variables}

The contribution of each covariate to early hemodialysis initiation was examined using logistic regression analyses (Table 4). The max BMI $\left(28.4 \mathrm{~kg} / \mathrm{m}^{2}\right)$ which was the cutoff value for early hemodialysis initiation identified in ROC analysis was entered in Model 1, and the max BMI (35.0 kg/ $\mathrm{m}^{2}$ ) which was the cutoff value for severe obesity requiring bariatric surgery was entered in Model 2. The analyses iden- 
Table 3. Comparison of characteristics of hemodialysis patients with hemodialysis initiated before age 50 versus age 50 or later

\begin{tabular}{|c|c|c|c|}
\hline \multirow[t]{2}{*}{ Variables } & \multicolumn{2}{|c|}{ Age at hemodialysis initiation } & \multirow[t]{2}{*}{$p$ value } \\
\hline & $\begin{array}{l}\geq 50 \text { years } \\
(N=579)\end{array}$ & $\begin{array}{l}<50 \text { years } \\
(N=145)\end{array}$ & \\
\hline Male, \% & 69.8 & 82.1 & $0.003^{*}$ \\
\hline Age, years & $70.2 \pm 9.1$ & $45.2 \pm 5.6$ & $<0.001$ \\
\hline Age at dialysis initiation, years & $67.7 \pm 9.3$ & $42.6 \pm 5.6$ & $<0.001$ \\
\hline Time on dialysis, years & $2.5 \pm 1.7$ & $2.6 \pm 1.7$ & 0.642 \\
\hline BMI at dialysis initiation, $\mathrm{kg} / \mathrm{m}^{2}$ & $22.3 \pm 3.5$ & $24.6 \pm 4.7$ & $<0.001$ \\
\hline $\mathrm{BMI}$ at dialysis initiation $\geq 35 \mathrm{~kg} / \mathrm{m}^{2}, \%$ & 0.3 & 5.5 & $<0.001^{*}$ \\
\hline Max BMI, kg/m² & $27.3 \pm 4.6$ & $30.6 \pm 6.4$ & $<0.001$ \\
\hline $\operatorname{Max} \mathrm{BMI} \geq 35 \mathrm{~kg} / \mathrm{m}^{2}, \%$ & 5.7 & 24.8 & $<0.001^{*}$ \\
\hline BMI difference, $\mathrm{kg} / \mathrm{m}^{2}$ & $5.0 \pm 3.5$ & $5.9 \pm 4.2$ & 0.034 \\
\hline Diabetes, \% & 53.9 & 55.2 & $0.852^{*}$ \\
\hline Sleep apnea syndrome, \% & 4.3 & 9.0 & $0.035^{*}$ \\
\hline Prevalence of atherosclerotic diseases, $\%$ & 34.9 & 32.4 & $0.625^{*}$ \\
\hline Myocardial infarction, \% & 7.4 & 6.2 & $0.721^{*}$ \\
\hline Coronary revascularization, $\%$ & 22.3 & 27.6 & $0.188^{*}$ \\
\hline Percutaneous transluminal angioplasty for PAD, \% & 5.7 & 3.4 & $0.404^{*}$ \\
\hline Limb amputation, $\%$ & 2.6 & 4.1 & $0.403^{*}$ \\
\hline Cerebral stroke, $\%$ & 12.3 & 5.5 & $0.017^{*}$ \\
\hline $\mathrm{PDR}, \%$ & 26.6 & 35.9 & $0.031^{*}$ \\
\hline Invasive treatment for diabetic retinopathy, $\%$ & 15.9 & 22.8 & $0.065^{*}$ \\
\hline Laser photocoagulation, $\%$ & 14.7 & 21.4 & $0.057^{*}$ \\
\hline Vitrectomy surgery, \% & 5.7 & 11.0 & $0.027^{*}$ \\
\hline
\end{tabular}

BMI difference was defined as max BMI minus BMI at hemodialysis initiation. Data are presented as mean \pm SD or percentage. Mann-Whitney U test. Max BMI, maximum lifetime body mass index; BMI, body mass index; $\mathrm{PAD}$, peripheral artery disease; SD, standard deviation; PDR, proliferative diabetic retinopathy. * Fisher's exact test was used to compare subjects in 2 groups.

Table 4. Bivariate logistic regression models for the association between hemodialysis initiation before age 50 and clinical variables

\begin{tabular}{|c|c|c|c|c|}
\hline \multirow[t]{2}{*}{ Variables } & \multicolumn{2}{|l|}{ Model 1} & \multicolumn{2}{|l|}{ Model 2} \\
\hline & ORs (95\% CIs) & $p$ value & ORs (95\% CIs) & $p$ value \\
\hline Male gender & $1.94(1.20-3.12)$ & 0.006 & $2.00(1.24-3.24)$ & 0.005 \\
\hline Sleep apnea syndrome & $1.56(0.748-3.26)$ & 0.235 & $1.48(0.688-3.19)$ & 0.315 \\
\hline Cerebral stroke & $0.378(0.175-0.817)$ & 0.013 & $0.383(0.176-0.837)$ & 0.016 \\
\hline Vitrectomy surgery & $1.74(0.905-3.36)$ & 0.097 & $1.83(0.932-3.60)$ & 0.079 \\
\hline Max $\mathrm{BMI} \geq 28.4 \mathrm{~kg} / \mathrm{m}^{2}$ & $2.57(1.75-3.79)$ & $<0.001$ & - & - \\
\hline $\operatorname{Max} \mathrm{BMI} \geq 35 \mathrm{~kg} / \mathrm{m}^{2}$ & - & - & $4.98(2.92-8.47)$ & $<0.001$ \\
\hline
\end{tabular}

Akaike's information criterion: 688.41 (model 1) and 678.36 (model 2); residual deviance: 676.41 (model 1) and 666.36 (Model 2); $p<0.001$ in both models. Max BMI, maximum lifetime body mass index; BMI, body mass index; ORs, odds ratios; CIs, confidence intervals. tified male gender, cerebral stroke, and either cutoff value of the max BMI as independent predictors for early hemodialysis initiation. Vitrectomy surgery for PDR also tended to contribute positively to early hemodialysis initiation, but the association was not significant.

Max BMI Influences Hemodialysis Initiation

\section{Discussion}

The present retrospective cross-sectional study, which examined the max BMI of hemodialysis patients, revealed that a history of obesity contributed to early hemodialysis 
initiation independent of diabetes. On the other hand, patients with early hemodialysis initiation showed a higher male ratio and higher prevalence of SAS and PDR. The following 2 max BMIs were extracted as independent contributors to hemodialysis initiation before age 50 in this study: $28.4 \mathrm{~kg} / \mathrm{m}^{2}$ which was the cutoff value identified in ROC analysis and $35 \mathrm{~kg} / \mathrm{m}^{2}$ which was the cutoff value for bariatric surgery. Additionally, the increase in the max BMI was also associated with prior coronary artery disease and PDR. These findings indicate the following 2 noteworthy lessons. First, it may be worthwhile for obese CKD patients to receive intensive weight reduction therapy promptly. Second, the max BMI should be used in risk assessment of ESRD in routine clinical practice. To the best of our knowledge, this is the first study to show an association between the max BMI and age of hemodialysis initiation in Japanese hemodialysis patients.

The biggest shortcoming of this study was that obesityrelated hypertension and dyslipidemia were not examined. In other words, it remains unclear whether the main cause of ESRD is a high max BMI per se, or obesity-related hypertension or dyslipidemia. If hypertension or dyslipidemia was the main underlying pathology of ESRD in this study, weight reduction therapy would have been unnecessary, and adequate control of blood pressure or lipid parameters by medications would have avoided the hemodialysis initiation. However, in this study population, obesity-related glomerulopathy (ORG), but not hypertension or dyslipidemia, is likely to have promoted CKD for the following 2 reasons. First, nephrosclerosis accounted for only $14.0 \%$ of the subjects' underlying kidney disease in this study. Second, some argue that obesity with ectopic adipose tissue is itself one of the pathogenic mediators that lead to the progression of CKD. It has been reported that high $\mathrm{BMI}$ is an independent risk factor for ESRD even after adjustment for atherosclerotic risks including smoking, hypertension, and diabetes [14]. In other words, obesity not only increases the risks for preexisting renal diseases but is also an independent risk factor of renal injury. ORG is defined pathologically as glomerulomegaly with focal segmental glomerulosclerosis [15].

In patients with ORG, various extra- and intrarenal pathophysiologies are involved in the progression of CKD $[16,17]$. First, cytokines derived from extrarenal visceral adipose tissue contributes to the pathogenesis of ORG. Obesity is a chronic low-grade inflammatory condition, in which adipose tissue serves as the source of cytokines. Visceral adipose tissue produces pro-inflammatory adipocytokines including tumor necrosis factor alpha and interleukin-6 which can induce renal inflammation. Furthermore, vascular endothelial growth factor (VEGF), a potent angiogenic and vascular permeability factor derived from adipose tissue [18], may contribute to the development of ORG. Increased serum concentration of VEGF is variably associated with metabolic syndrome or its components [19]. Besides, the expression of capillary growth promoting factors including VEGF is increased in ORG patients with glomerulomegaly [20]. Second, the role of intrarenal adipose tissue in patients with ORG has also been reported. Increased ectopic adipose tissue in the renal sinus, a cavity within the kidney, may limit the outflow of blood and lymph from the kidney, which would alter intrarenal physical forces and promote sodium reabsorption and arterial hypertension [21]. Additionally, such fatty kidney is involved in structural and functional changes of mesangial cells, podocytes, and proximal tubular cells, suggesting ORG as a maladaptive response to glomerular hyperfiltration and albuminuria [22]. These extra- and intrarenal pathophysiologies caused by obesity have also been supported by the previous reports indicating improved systemic vascular/ kidney functions after weight reduction therapy. For example, systemic arterial stiffness assessed by cardio-ankle vascular index has been shown to be high in subjects with obesity-related metabolic disorders and decrease by weight reduction therapy accompanied by visceral fat decrease [23]. Additionally, weight reduction therapy using formula diet improves renal function and proteinuria safely in patients with diabetic nephropathy and obesity [24]. Similarly, it has also been reported that bariatric surgery (laparoscopic sleeve gastrectomy) in Japanese patients with severe obesity achieves relatively long-term improvement in renal function [25]. Besides, there is also a proposal to modify the BMI criterion for metabolic surgery from 35 to $27 \mathrm{~kg} / \mathrm{m}^{2}$ or higher for uncontrolled diabetes patients [26,27], and many young CKD patients are expected to benefit from this expanded indication. When extrapolating the above findings to the present study, the hemodialysis patients who were obese in the past could have been able to avoid or postpone hemodialysis initiation if they had controlled obesity in the past by undergoing weight reduction therapy. On the other hand, there is not yet sufficient data to support statements such as that intensive weight reduction therapy is effective to avoid hemodialysis initiation [28], so further verification is needed.

In the present study, as mentioned above, a high max BMI contributed to early hemodialysis initiation probably due to ORG, independent of diabetes. On the other hand, $\mathrm{PDR}$, especially PDR requiring vitrectomy, was also asso- 
ciated with a high max BMI and early hemodialysis initiation in univariate analyses. This finding suggests that excess adipose tissue-derived VEGF may promote retinal lesions. VEGF is known to be a principal mediator of diabetic retinopathy, capable of inducing the changes observed in PDR and macular edema [29]. Furthermore, Shiba et al. [30] have reported that intravitreal injection of anti-VEGF drugs improves not only the pathophysiology of retinal lesions but also renal function and systemic arterial stiffness. Early weight reduction therapy for obese patients therefore may contribute to avoid hemodialysis and vision impairment through reducing VEGF expression.

In Table 1, the male ratio increased with increasing tertiles of the max BMI, indicating that men had a relatively higher max BMI. Indeed, men had a significant higher max BMI than that of women $\left(28.5 \mathrm{vs} .26 .7 \mathrm{~kg} / \mathrm{m}^{2}\right)$ in the present study (data not shown). However, male gender as a risk factor for early hemodialysis initiation was independent of obesity, and this finding is consistent with the fact that male gender is an independent coronary risk factor [31].

In Table 3, patients with hemodialysis initiation after age 50 had a significantly higher prevalence of cerebral stroke than patients with early hemodialysis initiation, which may be related to their higher mean age (70.2 vs. 45.2 years). Furthermore, in the logistic model presented in Table 4, the low contribution of cerebral stroke to hemodialysis initiation before age $50(\mathrm{OR}=0.375, p<0.05)$ may also be due to the intra-class correlation of cerebral stroke with aging.

Limitations of this study include the lack of analyses in some potential confounders such as hypertension, dyslipidemia, hyperuricemia, alcohol consumption, and smoking status. Furthermore, the cross-sectional nature of this study does not allow determination of the time course to confirm the causal relationship between the max BMI and hemodialysis initiation. Additionally, our findings may not be generalized to other ethnic groups. From these points of view, a large-scale prospective cohort study, including ethnically different population such as Caucasian that also covers the age at the max BMI is needed.

\section{Conclusion}

This study in hemodialysis patients showed that a high BMI at younger ages contributed to early hemodialysis initiation, independent of diabetes. Furthermore, higher prevalence of vascular complications such as coronary artery disease and PDR was observed in patients with younger age of hemodialysis initiation and higher BMI. Considering previous reports that weight reduction in obese patients improves renal function, weight reduction in young CKD patients with obesity might be effective to avoid or postpone hemodialysis and development of vascular complications, especially PDR.

\section{Acknowledgments}

The authors are grateful to Dr. Atsuhito Saiki (Center of Diabetes, Endocrine and Metabolism, Toho University Sakura Medical Center, Chiba, Japan) for valuable advice and wish to thank Chikako Taniguchi (Division of Nursing, Tokatsu Clinic Hospital), Erika Sasaki (Division of Nursing, Tokatsu Clinic Mirai), Kyomi Hasegawa (Division of Nursing, Tokatsu Clinic Abiko), Hatsuhiko Tobari (Division of Nursing, Tokatsu Clinic Matsudo), Soko Ueno (Division of Nursing, Tokatsu Clinic Kasiwa), Kiyomi Ichikawa (Division of Nursing, Tokatsu Clinic Shin-Matsudo), Sanae Yoshioka (Division of Nursing, Tokatsu Clinic Koiwa), Hironori Aoki (Division of Nursing, Tokatsu Clinic Yabashira), Chihiro Matsumoto, Masami Osaka, Manami Nihei, Keiko Miyamoto, and Natsuko Hiroki (Division of Nutrition, Mihama Hospital) for collection and assembly of data.

\section{Statement of Ethics}

The protocol of the study was prepared in accordance with the Declaration of Helsinki and was approved by the Ethics Committee of Tokatsu Clinic Hospital (No. 2016-04) and Mihama Hospital (No. 16-002). Written informed consent was obtained from all of the participants.

\section{Conflict of Interest Statement}

The authors declared no conflict of interest.

\section{Funding Sources}

This study was supported by a grant for research on intractable diseases from Ministry of Health, Labour and Welfare of Japan (H28-nanji-ippan-014).

\section{Author Contributions}

N.A., K.S., E.S., T.S., and M.M. contributed to collection and/ or assembly of data. A.O. and D.N. contributed to the research concept and design, collection and/or assembly of data, data analysis, and writing of the article. K.S. and I.T. contributed to data interpretation and critical revision of the manuscript. All the authors approved the version to be published.

\section{Data Availability Statement}

The data that support the findings of this study are not publicly available due to their containing information that could compromise the privacy of research participants. Further enquiries can be directed to the corresponding author. 


\section{References}

1 Adams KF, Schatzkin A, Harris TB, Kipnis V, Mouw T, Ballard-Barbash R, et al. Overweight, obesity, and mortality in a large prospective cohort of persons 50 to 71 years old. N Engl J Med. 2006;355(8):763-78.

2 Ejerblad E, Fored CM, Lindblad P, Fryzek J, McLaughlin JK, Nyrén O. Obesity and risk for chronic renal failure. J Am Soc Nephrol. 2006; 17(6):1695-702.

3 Swinburn BA, Sacks G, Hall KD, McPherson K, Finegood DT, Moodie ML, et al. The global obesity pandemic: shaped by global drivers and local environments. Lancet. 2011; 378(9793):804-14.

4 Kramer HJ, Saranathan A, Luke A, DurazoArvizu RA, Guichan C, Hou S, et al. Increasing body mass index and obesity in the incident ESRD population. J Am Soc Nephrol. 2006;17(5):1453-9.

5 GBD 2019 Blindness And Vision Impairment Collaborators; Vision Loss Expert Group Of The Global Burden Of Disease Study. Causes of blindness and vision impairment in 2020 and trends over 30 years, and prevalence of avoidable blindness in relation to VISION 2020: the right to sight: an analysis for the global burden of disease study. Lancet Glob Health. 2020;S2214-109X(20)30489-7.

6 Howell CR, Fontaine K, Ejima K, Ness KK, Cherrington A, Mehta T. Maximum lifetime body mass index and mortality in Mexican American adults: the National Health and Nutrition Examination Survey III (19881994) and NHANES 1999-2010. Prev Chronic Dis. 2017;14:E67.

7 Committee of the Japan Diabetes Society on the Diagnostic Criteria of Diabetes Mellitus, Seino Y, Seino Y, Nanjo K, Tajima N, Kadowaki T, et al. Report of the committee on the classification and diagnostic criteria of diabetes mellitus. J Diabetes Investig. 2010;1(5): $212-28$.

8 Japan Society for the Study of Obesity. Diagnostic criteria for obesity. J Jpn Soc Study Obes. 2011:17:1-78.

9 Kanda Y. Investigation of the freely available easy-to-use software "EZR" for medical statistics. Bone Marrow Transplant. 2013;48(3): $452-8$.
10 Nitta K, Goto S, Goto S, Masakane I, Hanafusa N, Taniguchi $\mathrm{M}$, et al. Annual dialysis data report for 2018, JSDT renal data registry: survey methods, facility data, incidence, prevalence, and mortality. Ren Replace Ther. 2020;6(1):41.

11 Ministry of Health Labour and Welfare of Japan. National health and nutrition survey report; 2018. Available from: https://www. mhlw.go.jp/stf/seisakunitsuite/bunya/kenkou_iryou/kenkou/eiyou/h 30 - hou koku_00001.html.

12 Youden WJ. Index for rating diagnostic tests. Cancer. 1950;3(1):32-5.

13 Sasaki A, Wakabayashi G, Yonei Y. Current status of bariatric surgery in Japan and effectiveness in obesity and diabetes. J Gastroenterol. 2014;49(1):57-63.

14 Hsu CY, McCulloch CE, Iribarren C, Darbinian J, Go AS. Body mass index and risk for end-stage renal disease. Ann Intern Med. 2006;144(1):21-8.

$15 \mathrm{Xu} \mathrm{T}$, Sheng Z, Yao L. Obesity-related glomerulopathy: pathogenesis, pathologic, clinical characteristics and treatment. Front Med. 2017;11(3):340-8.

16 Xu H, Barnes GT, Yang Q, Tan G, Yang D, Chou CJ, et al. Chronic inflammation in fat plays a crucial role in the development of obesity-related insulin resistance. J Clin Invest. 2003;112(12):1821-30.

17 Harkins JM, Moustaid-Moussa N, Chung YJ, Penner KM, Pestka JJ, North CM, et al. Expression of interleukin- 6 is greater in preadipocytes than in adipocytes of 3T3-L1 cells and C57BL/6J and ob/ob mice. J Nutr. 2004; 134(10):2673-7.

18 Ferrara N, Davis-Smyth T. The biology of vascular endothelial growth factor. Endocr Rev. 1997;18(1):4-25.

19 Miyazawa-Hoshimoto S, Takahashi K, Bujo $\mathrm{H}$, Hashimoto N, Saito Y. Elevated serum vascular endothelial growth factor is associated with visceral fat accumulation in human obese subjects. Diabetologia. 2003;46(11): 1483-8.

20 Tsuboi N, Okabayashi Y, Shimizu A, Yokoo T. The renal pathology of obesity. Kidney Int Rep. 2017;2(2):251-60.

21 Chughtai HL, Morgan TM, Rocco M, Stacey B, Brinkley TE, Ding J, et al. Renal sinus fat and poor blood pressure control in middleaged and elderly individuals at risk for cardiovascular events. Hypertension. 2010;56(5): 901-6.
22 de Vries AP, Ruggenenti P, Ruan XZ, Praga M, Cruzado JM, Bajema IM, et al. Fatty kidney: emerging role of ectopic lipid in obesityrelated renal disease. Lancet Diabetes Endocrinol. 2014;2(5):417-26.

23 Nagayama D, Endo K, Ohira M, Yamaguchi T, Ban N, Kawana H, et al. Effects of body weight reduction on cardio-ankle vascular index (CAVI). Obes Res Clin Pract. 2013 Mar; 7(2):e139-45.

24 Saiki A, Nagayama D, Ohhira M, Endoh K, Ohtsuka M, Koide N, et al. Effect of weight loss using formula diet on renal function in obese patients with diabetic nephropathy. Int J Obes. 2005;29(9):1115-20.

25 Saiki A, Yamaguchi T, Tanaka S, Sasaki A, Naitoh T, Seto Y, et al. Background characteristics and postoperative outcomes of insufficient weight loss after laparoscopic sleeve gastrectomy in Japanese patients. Ann Gastroenterol Surg. 2019;3(6):638-47.

26 Kasama K, Mui W, Lee WJ, Lakdawala M, Naitoh T, Seki Y, et al. IFSO-APC consensus statements 2011. Obes Surg. 2012;22(5):67784.

27 Schauer PR, Bhatt DL, Kirwan JP, Wolski K, Aminian A, Brethauer SA, et al. Bariatric surgery versus intensive medical therapy for diabetes - 5-year outcomes. N Engl J Med. 2017; 376(7):641-51.

28 Conley MM, McFarlane CM, Johnson DW, Kelly JT, Campbell KL, MacLaughlin HL. Interventions for weight loss in people with chronic kidney disease who are overweight or obese. Cochrane Database Syst Rev. 2021; 3(3):CD013119.

29 Aiello LP, Wong JS. Role of vascular endothelial growth factor in diabetic vascular complications. Kidney Int Suppl. 2000;77:S113-9.

30 Shiba T, Takahashi M, Yoshida I, Taniguchi H, Matsumoto T, Hori Y. Arteriosclerotic changes after intravitreal injections of antivascular endothelial growth factor drugs in patients with exudative age-related macular degeneration. Ophthalmologica. 2016;235(4): 225-32.

31 Nishimura K, Okamura T, Watanabe M, Nakai M, Takegami M, Higashiyama A, et al. Predicting coronary heart disease using risk factor categories for a Japanese urban population, and comparison with the framingham risk score: the suita study. J Atheroscler Thromb. 2014;21(8):784-98. 